\title{
Valsalva-Like Retinopathy Spontaneously Occurred after Ocular Massage
}

\author{
Keiko Uchida Masayuki Takeyama Masahiro Zako \\ Department of Ophthalmology, Aichi Medical University, Nagakute, Japan
}

\section{Key Words}

Neodymium-doped yttrium-aluminium-garnet laser membranotomy - Ocular massage . Valsalva-like retinopathy

\begin{abstract}
Purpose: The aim of this study is to describe a case of Valsalva-like retinopathy that occurred after ocular massage. Case Presentation: A healthy 44-year-old Japanese female had massaged her eye with strong pressure several times. Subsequently, she noticed a loss in the left central vision. A left-eye fundus examination showed a dense preretinal hemorrhage located under the internal limiting membrane at the posterior pole and a mild vitreous hemorrhage. We performed a neodymium-doped yttrium-aluminium-garnet laser membranotomy to perforate the internal limiting membrane. Her best-corrected visual acuity improved from 0.01 to 1.0. No retinal vascular abnormalities in the macular area were found. Conclusion: Ocular massage can cause Valsalva-like retinopathy.

(c) 2015 S. Karger AG, Basel
\end{abstract}

\section{Introduction}

Valsalva retinopathy is defined as preretinal hemorrhage secondary to a sudden increase in central venous pressure caused by heavy lifting, straining during defecation, physical exercise, childbirth labor, coughing, or vomiting [1-3]. The accepted mechanism is that elevated central venous pressure, secondary to an increase in intra-abdominal or intrathoracic pressure, causes the retinal capillaries to rupture. Here, we describe a case of Valsalva-like retinopathy, suspected to be caused by ocular massage, without central venous pressure elevation. 
Uchida et al.: Valsalva-Like Retinopathy Spontaneously Occurred after Ocular Massage

\section{Case Presentation}

A 44-year-old healthy Japanese female experienced pain in her left eye when she was relaxed and watching television. She massaged her eyes with strong pressure several times with her fingers. Subsequently, she noticed a sudden loss in the left central vision. The next day, she visited our hospital. When questioned, she could not remember having done anything that might induce Valsalva retinopathy.

Upon examination, her left eye had a best-corrected visual acuity of 0.01 , intraocular pressure (IOP) was $17 \mathrm{~mm} \mathrm{Hg}$, and the cornea and lens were clear. A left fundus examination showed a dense preretinal hemorrhage at the posterior pole (fig. 1a). The preretinal hemorrhage appeared to be located under the internal limiting membrane (ILM) due to the reflection of the surface of the preretinal hemorrhage, and this was confirmed by optical coherence tomography (fig. 1b). A mild vitreous hemorrhage was also observed. Fluorescein and indocyanine green angiography showed no retinal vascular abnormality, although the posterior pole was veiled by the preretinal hemorrhage (fig. 1c, d). In her right eye, no abnormality was found. The blood examination including complete blood counts and clotting parameters were within normal limits.

During her first visit to our hospital, we could not perform a neodymium-doped yttriumaluminium-garnet laser membranotomy to perforate the ILM due to interference by the vitreous hemorrhage. Five days after the first visit, the vitreous hemorrhage had decreased and we successfully performed the neodymium-doped yttrium-aluminium-garnet laser membranotomy (power setting, $5.4 \mathrm{~mJ}$ ) using Goldmann's three-mirror contact lens. The preretinal hemorrhage immediately moved to the vitreous cavity through the open hole in the ILM. Two days after the treatment, the best-corrected visual acuity improved to 1.0, but a small amount of the vitreous hemorrhage and residual preretinal hemorrhage were observed at the posterior pole (fig. 2a). The optical coherence tomography image showed remaining ILM elevation (fig. 2b). One month after the treatment, we performed another fluorescein and indocyanine green angiography, and it demonstrated no retinal vascular abnormalities in the macular area (fig. 2c, d).

\section{Discussion}

The precise mechanism that induced Valsalva-like retinopathy in our patient's left eye was unclear. We speculate that the Valsalva-like retinopathy occurred due to the following mechanism. First, the compression of the ocular massage deformed both the eye globe and the retinal structures in the macular area. This pressure raised the IOP for a short time. Second, the massage caused a shear stress forming between the retinal capillaries and the surrounding structures on the retinal surface. This shear stress may occur when the posterior vitreous undergoes a lateral transition, even in a patient without a posterior vitreous detachment. Finally, the shear stress stretched the fragile retinal capillaries, which then ruptured in the macular area and bled into the space below the ILM, inside and outside the vitreous cavity.

This report showed that Valsalva-like retinopathy may be induced by local factors that do not involve a systemic factor. Moshfeghi et al. [4] described a similar preretinal hemorrhage, or Valsalva-like retinopathy, after performing laser in situ keratomileusis, a procedure that requires deformation of the eye globe. During this procedure, the IOP was raised above $85 \mathrm{~mm} \mathrm{Hg}$ for a short time [5]. That case, in conjunction with our case, suggests that Valsal- 
Uchida et al.: Valsalva-Like Retinopathy Spontaneously Occurred after Ocular Massage

va-like retinopathy could be induced by concurrently deforming the eye globe and raising the IOP for a short time.

\section{Disclosure Statement}

The authors have no conflicts of interest to declare.

\section{References}

1 Duane TD: Valsalva hemorrhagic retinopathy. Trans Am Ophthalmol Soc 1972;70:298-313.

-2 Eneh A: Valsalva hemorrhagic retinopathy during labour: a case report and literature review. Can J Ophthalmol 2013;48:145-147.

3 Chen Y, Zhao M, Zhou P: Traumatic chorioretinopathies; in Ryan SJ (ed): Retina, ed 5. New York, Elsevier, 2013, vol 2, pp 1564-1570.

-4 Moshfeghi AA, Harrison SA, Reinstein DZ, Ferrone PJ: Valsalva-like retinopathy following hyperopic laser in situ keratomileusis. Ophthalmic Surg Lasers Imaging 2006;37:486-488.

-5 Conway ML, Wevill M, Benavente-Perez A, Hosking SL: Ocular blood-flow hemodynamics before and after application of a laser in situ keratomileusis ring. J Cataract Refract Surg 2010;36:268-272. 


\section{Case Reports in \\ Ophthalmology}

\begin{tabular}{l|l}
\hline \multicolumn{2}{l}{ Case Rep Ophthalmol 2015;6:88-92 } \\
\hline DOI: 10.1159/000380877 & $\begin{array}{l}\text { @ 2015 S. Karger AG, Basel } \\
\text { www.karger.com/cop }\end{array}$ \\
\hline
\end{tabular}

Uchida et al.: Valsalva-Like Retinopathy Spontaneously Occurred after Ocular Massage
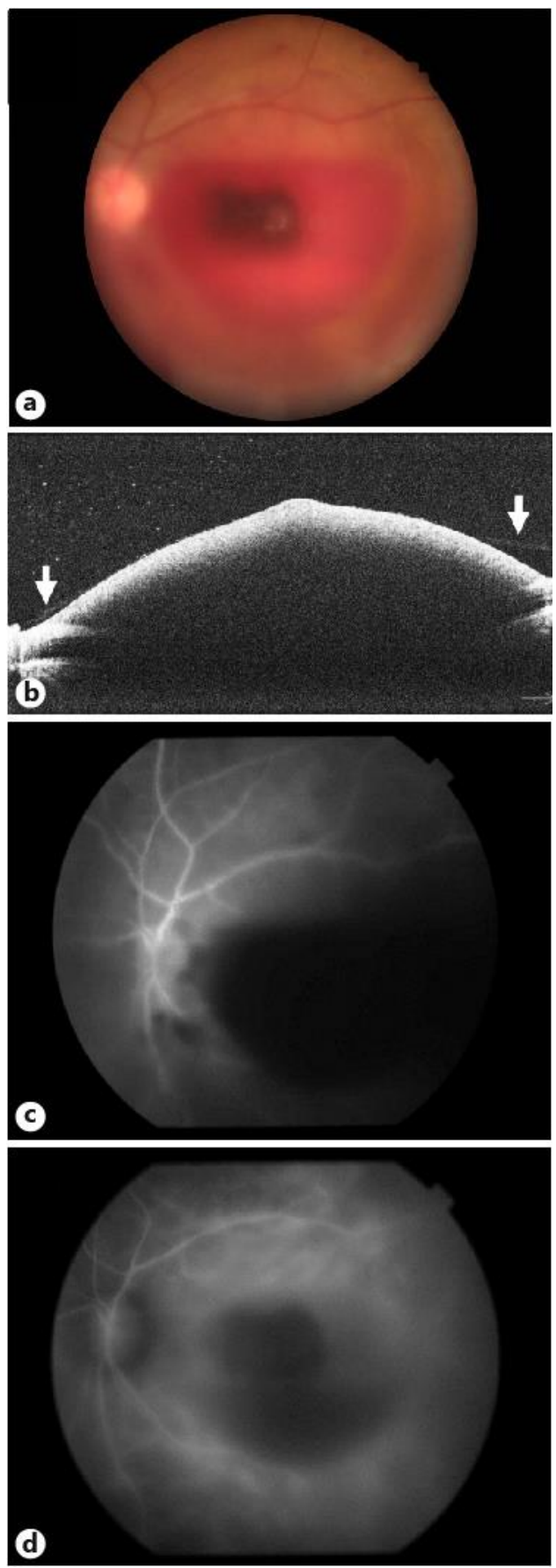

Fig. 1. Preretinal hemorrhage in an eye that exhibited Valsalva-like retinopathy. a Fundus photograph shows the hemorrhage over the central section of the eye. b Optical coherence tomography image shows the preretinal hemorrhage that was located under the ILM. The posterior hyaloid membrane was demonstrated above the preretinal hemorrhage (arrows). c Fluorescein angiography. d Indocyanine green angiography. No visible retinal vascular abnormality was observed; however, the posterior pole was veiled due to the preretinal hemorrhage. 


\section{Case Reports in \\ Ophthalmology}

\begin{tabular}{l|l}
\hline \multicolumn{2}{l}{ Case Rep Ophthalmol 2015;6:88-92 } \\
\hline DOI: 10.1159/000380877 & $\begin{array}{l}\text { @ 2015 S. Karger AG, Basel } \\
\text { www.karger.com/cop }\end{array}$ \\
\hline
\end{tabular}

Uchida et al.: Valsalva-Like Retinopathy Spontaneously Occurred after Ocular Massage
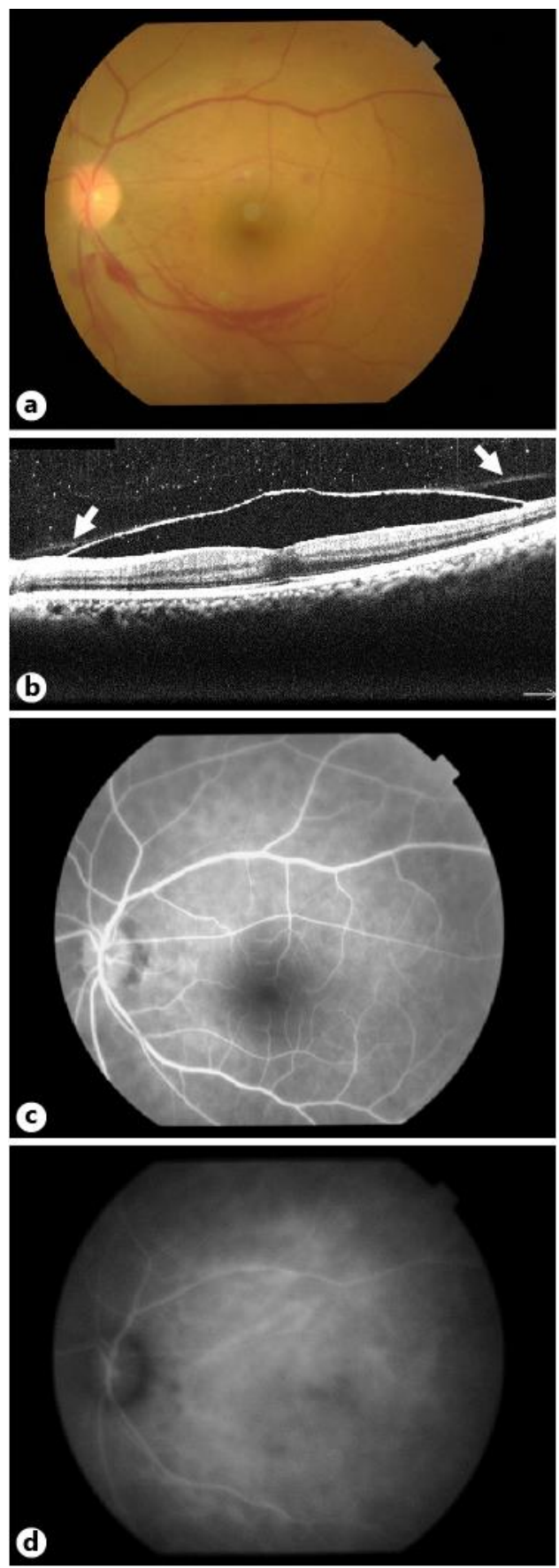

Fig. 2. Retinopathy was resolved with a membranotomy. a Fundus photograph taken 2 days after membranotomy. b Optical coherence tomography image shows the posterior hyaloid membrane (arrows) and the remaining ILM elevation. c Fluorescein angiography. d Indocyanine green angiography. No retinal vascular abnormality was demonstrated. 